\title{
AML with Renal Infiltration Manifesting as Acute Renal Failure, Diagnosed with FDG-PET CT Scan: Case Report
}

\author{
Chauhan P'*, Gupta A ${ }^{1}$, Ora $\mathbf{M}^{2}$, Agrawal S ${ }^{1}$ and \\ Nityanand $\mathbf{S}^{1}$ \\ ${ }^{1}$ Department of Haematology, SGPGIMS, Lucknow (UP), \\ India \\ ${ }^{2}$ Department of Nuclear Medicine, SGPGIMS, Lucknow \\ (UP), India \\ *Corresponding author: Priyanka Chauhan, \\ Department of Haematology, I block, SGPGIMS, \\ Raebareli Road, 226014, Lucknow, India
}

Received: June 21, 2021; Accepted: July 15, 2021; Published: July 22, 2021

\begin{abstract}
Extramedullary disease in acute myeloid leukemia is a known phenomenon with reported incidence of 2.5-9.1\%. However, acute kidney injury due to direct infiltration of malignant cells is reported in only $1 \%$ cases of acute leukemia. We report a case of acute myeloid leukemia who developed acute kidney failure at presentation, was diagnosed with renal and pancreatic infiltration by FDG-PET scan and was treated successfully with hypomethylating agent and venetoclax. PET-CT scan can be a non-invasive modality for diagnosing extramedullary disease in acute myeloid leukemia and monitoring of response to therapy in these cases. Early initiation of anti-leukemia therapy in our case lead to complete metabolic response with normalization of the renal functions.
\end{abstract}

Keywords: Acute kidney injury; Acute myeloid leukemia; FDG-PET CT scan

\section{Abbreviation}

AML: Acute Myeloid Leukaemia; AKI: Acute Kidney Injury; PETCT: Positron Emission Tomography and Computed Tomography; HMA: Hypomethylating Agent; GMI: Galactomannan Index; CECT: Contrast Enhanced Computed Tomography; TLS: Tumour Lysis Syndrome

\section{Case Presentation}

Acute Kidney Injury (AKI) is the most common renal complication in haematological malignancies [1-5]. Different mechanisms leading to acute kidney injury in haematological malignancies includes tumour lysis syndrome, hypoperfusion, acute tubular necrosis and direct renal infiltration [6]. Acute renal failure arising from direct renal infiltration of malignant cells has been reported in only $1 \%$ cases acute leukaemia [7,8]. We report a case of Acute Myeloid Leukaemia (AML) with waxing and waning course of AKI diagnosed with the help of FDG PET-CT scan and managed successfully with Hypomethylating Agent (HMA) + Venetoclax based therapy.

50 -year-old male patient with history of type 2 diabetes mellitus for 6 years, hypertension for 5 years and anxiety disorder for 1 year, presented to our hospital with complaints of fever and generalized weakness for 20 days. Patient required 4 units of blood transfusions during this period. No history of rashes over body, yellowish discolouration of eyes, cough, bleeding from any site, decreased urine output, swelling over any area of body or facial puffiness.

Examination was unremarkable except for moderate pallor. Baseline investigations are enlisted in Table 1.

Patient was diagnosed as AML low risk, had fever at presentation, Galactomannan Index (GMI) was positive and Contrast Enhanced CT scan (CECT) thorax showed evidence of fungal pneumonia, patient was started on liposomal amphotericin $b$ for probable fungal pneumonia (ECIL criteria), post 2 weeks of amphotericin, patient became afebrile. In view of poor performance status (ECOG-2) and co-morbidities, was planned for hypomethylating agent + venetoclax based therapy. Patient received decitabine for 5 days @20mg $/ \mathrm{m}^{2}$, Venetoclax was started on day $6 @ 100 \mathrm{mg}$ per day, after first dose of Venetoclax patient's urine output reduced to $0.7 \mathrm{ml} / \mathrm{kg} / \mathrm{hr}$ and serum creatinine increased to $3.8 \mathrm{mg} / \mathrm{dl}$ (Table 1). Nephrology opinion was sought for AKI and patient was investigated for the possible aetiology. Hypovolemia was ruled out, as there was no history suggestive of diarrhoea, vomiting or blood loss, Fractional excretion of $\mathrm{Na}(\mathrm{FeNa})$ was consistent with renal AKI. Laboratory Tumour Lysis Syndrome (TLS) parameters showed hyperkalaemia, hyperphosphatemia, and mild hypocalcaemia (Table 1). Sepsis work up including procalcitonin, urine cultures and blood cultures were all-negative. The differentials of TLS vs. Acute tubular necrosis were kept. All nephrotoxic medications including amphotericin were stopped considering a possibility of acute tubular necrosis and patient was started on oral voriconazole for fungal pneumonia. Ultrasound abdomen showed bilateral bulky kidneys with no evidence of hydronephrosis or any renal mass. The next day patient's renal function further deteriorated, and he developed features of volume overload with hyperkalaemia and had to be haemodialyzed, after 3 cycles of haemodialysis the renal parameters stabilised, and patient was continued on conservative management with which his kidney function improved over a period of 10 days and his serum creatinine stabilised between $1.8-2.0 \mathrm{mg} / \mathrm{dl}$. After interruption of therapy for 15 days patient's kidney functions again worsened and rapidly progressed to anuria and again required 2 cycles of haemodialysis. No nephrotoxic drugs were administered during this period and TLS parameters were within normal ranges in this episode. Renal biopsy could not be attempted due to poor clinical status and thrombocytopenia. An FDG-PET-CT scan was planned to look for renal infiltration with AML, PET-CT revealed metabolically active, enlarged kidney and pancreas-likely disease involvement (Figure 1A, 2A and 2B). Patient was immediately started on $2^{\text {nd }}$ cycle decitabine with Venetoclax with adequate hydration and tumour lysis prophylaxis, after a period of 10 days of therapy, his renal profile normalized, and patient was continued on decitabine + Venetoclax. Post 4 cycles of HMA + Venetoclax, bone marrow showed morphological remission and PET-CT showed complete 
Table 1: Investigations at baseline, during $\mathrm{AKI}^{*}$ episodes and post therapy.

\begin{tabular}{|c|c|c|c|c|}
\hline Parameter & Baseline & $A K I^{*}$-first episode & $\begin{array}{l}\text { AKI*-Second } \\
\text { episode }\end{array}$ & Post 4 cycles HMA + Venetoclax \\
\hline $\mathrm{Hb}(\mathrm{g} / \mathrm{dl})$ & 7.1 & 7.9 & 6.3 & 8.8 \\
\hline TLC $\left({ }^{*} 1000 / u l\right)$ & 46.3 & 1.3 & 1 & 4.3 \\
\hline DLC & N1L9Blasts90 & N21L76M3 & N48L44M6E2 & N64L28M8 \\
\hline $\begin{array}{l}\text { Platelet }\left({ }^{*} 1000 /\right. \\
\left.\mathrm{mm}^{3}\right)\end{array}$ & 55 & 20 & 72 & 184 \\
\hline S.creatinine $(\mathrm{mg} / \mathrm{dl})$ & 0.8 & 3.8 & 5.6 & 1.2 \\
\hline BUN (u/L) & 12 & 80 & 104 & 25 \\
\hline S.uric acid (mg/dl) & 3.5 & 5.1 & 7.2 & NA \\
\hline $\begin{array}{l}\text { S.Phosphorous } \\
\text { (mg/dl) }\end{array}$ & 5 & 6 & 6.3 & NA \\
\hline $\begin{array}{l}\text { S.calcium-ionic } \\
(\mathrm{mg} / \mathrm{dl})\end{array}$ & 4.8 & 4.3 & 4.5 & 4.6 \\
\hline $\begin{array}{l}\text { S.potassium } \\
(\mathrm{mmol} / \mathrm{L})\end{array}$ & 4.3 & 6 & 5.55 & 3.6 \\
\hline $\mathrm{LDH}(\mathrm{U} / \mathrm{L})$ & 125 & 222 & 113 & 210 \\
\hline $\mathrm{GMI}^{*}$ & 0.93 & 0.7 & NA & NA \\
\hline $\begin{array}{l}\text { Procalcitonin (ng/ } \\
\mathrm{ml})\end{array}$ & 0.14 & 0.73 & 0.41 & NA \\
\hline Urine routine & WNL & $\begin{array}{c}\text { Negative for WBC, RBC, cast or } \\
\text { sediment }\end{array}$ & WNL & NA \\
\hline Urine culture & Sterile & Sterile & Sterile & NA \\
\hline $\mathrm{FeNa}$ & NA & & & \\
\hline $\begin{array}{l}\text { Bone marrow } \\
\text { aspirate }\end{array}$ & $\begin{array}{l}\text { Smear shows } 85 \% \text { blasts with Auer rods and } \\
\text { MPO positive }\end{array}$ & NA & NA & $\begin{array}{l}\text { Hemodiluted aspirate with no excess } \\
\text { of blasts }\end{array}$ \\
\hline $\begin{array}{l}\text { Bone marrow } \\
\text { Biopsy }\end{array}$ & $\begin{array}{l}\text { Hypercellular marrow with near total } \\
\text { replacement by blasts }\end{array}$ & NA & NA & $\begin{array}{l}\text { Mildly hypocellular marrow with no } \\
\text { excess of blasts }\end{array}$ \\
\hline Flow cytometry & $\begin{array}{l}\text { 84\%blasts- positive for MPO/CD13/CD33/ } \\
\text { CD117/CD38 }\end{array}$ & NA & NA & NA \\
\hline Cytogenetics & $46, X Y$ & NA & NA & NA \\
\hline Mutational Profile & $\begin{array}{l}\text { NPM1 + by Multiplex PCR and capillary } \\
\text { electrophoresis }\end{array}$ & NA & NA & NA \\
\hline
\end{tabular}

*Acute kidney injury; ${ }^{\$}$ Hypomethylating agent; "Galactomannan index.

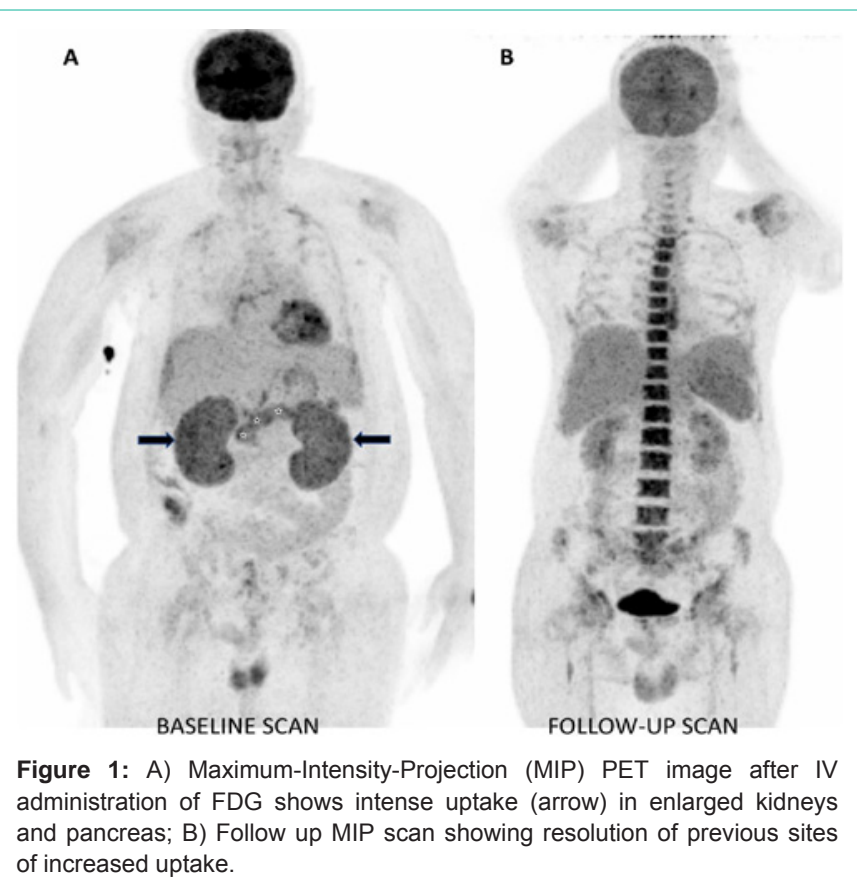

resolution of previous lesions (Figure 1B, 2C and 2D) thereby proving the causal association.

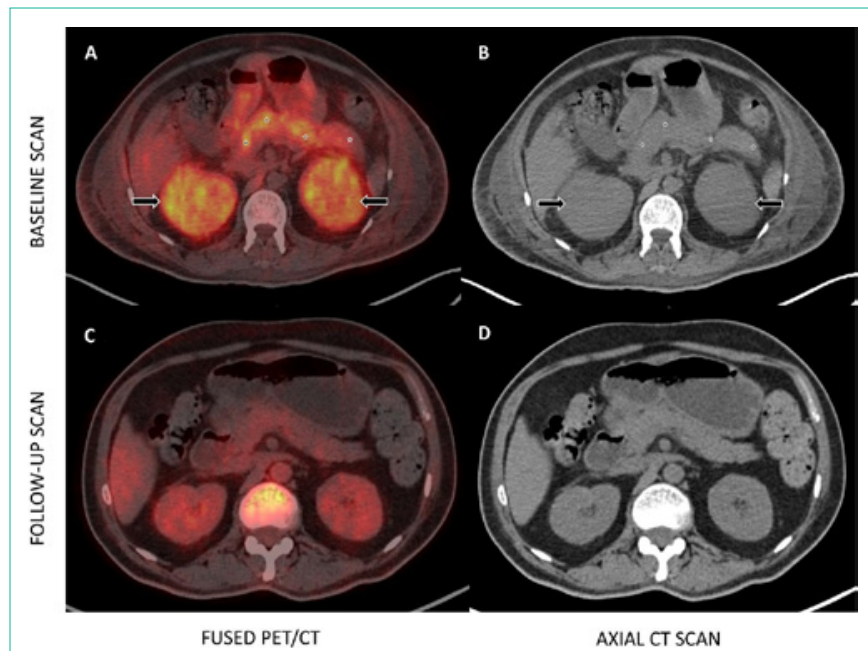

Figure 2: A, B) Axial fused PET/CT and CT scan images showing (arrows) bilateral enlarged kidneys and bulky pancreas with increased parenchymal uptake (SUVmax-4.27); C, D) Follow up scan showing complete metabolic resolution of previously seen lesions.

\section{Discussion/ Conclusion}

This case report highlights the rare presentation of AML with renal infiltration mimicking tumour lysis syndrome at initial presentation 
and demonstrates the importance of non-invasive PET-CT imaging in cases where renal biopsy cannot be performed due to poor clinical status of patient and associated complications of invasive procedure. In a prospective PETAML trial [9] FDG-PET/CT was demonstrated a useful and safe tool to detect EM AML with a high sensitivity and specificity of $77 \%$ and $97 \%$, respectively. In this study PET-CT was a more sensitive modality to detect extramedullary disease as compared to clinical and histopathological studies and to monitor response to therapy. In our patient the findings could not be confirmed on biopsy, but complete metabolic response post therapy proves disease involvement. Rapid diagnosis of this rare presentation and early AML specific therapy is the key to restore renal function.

\section{References}

1. Liu PI, Ishimaru T, McGregor DH, Okada H, Steer A. Autopsy study of granulocytic sarcoma (chloroma) in patients with myelogenous leukemia, Hiroshima-Nagasaki 1949-1969. Cancer. 1973; 31: 948-955.

2. Neiman RS, Barcos M, Berard C, et al. Granulocytic sarcoma: clinicopathologic study of 61 biopsied cases. Cancer. 1981; 48: 1426-1437.
3. Wiernik PH, Serpick AA. Granulocytic sarcoma (chloroma). Blood. 1970; 35 361-369.

4. Randy L Luciano and Ursula C Brewster. Kidney Involvement in Leukemia and Lymphoma. Advances in Chronic Kidney Disease. 2014; 21: 27-35.

5. Sae Aratani et al. Primary Tumor Infiltration and Severe Acute Kidney Injury in Patients with Acute Myeloblastic Leukemia. J Nippon Med Sch. 2020; 87: 43-18.

6. Khai Yang Ooi, et al. Infiltrative acute myeloid leukaemia as a cause of acute kidney injury. Clin Kidney J. 2013; 6: 448-449.

7. Xiao JC, Walz-Mattmüller $R$, Ruck $P$, et al. Renal involvement in myeloproliferative and lymphoproliferative disorders. A study of autopsy cases. Gen Diagn Pathol. 1997; 142: 147-153.

8. Duda J, Zoger S. Presentation of M4 acute myeloid leukemia in anuric renal failure with hyperuricemia and enlarged kidneys. J Pediatr Hematol Oncol. 2002; 24: 55-58.

9. Friedrich Stölzel, Tors Lüer et al. The prevalence of extramedullary acute myeloid leukemia detected by 18FDG-PET/CT: final results from the prospective PETAML trial. Haematol. 2020; 105: 1552-1558. 